\title{
Highly Luminescent Encapsulated Narrow Bandgap Polymers Based on Diketopyrrolopyrrole
}

\author{
Anastasia Leventis ${ }^{1 \ddagger}$, Jeroen Royakkers ${ }^{1 \ddagger}$, Alexandros Rapidis ${ }^{2}$, Niall Goodeal ${ }^{1}$, Merina Corpinot $^{3}$, Jar- $^{-}$ \\ vist M. Frost ${ }^{4}$, Dejan-Krešimir Bučar ${ }^{3}$, Matt Blunt ${ }^{3}$, Franco Cacialli ${ }^{2}$, Hugo Bronstein ${ }^{1 *}$ \\ 1 Department of Chemistry, University of Cambridge, Lensfield Rd, Cambridge CB2 1EW \\ 2 Department of Physics and Astronomy and LCN, University College London, Gower Street, London WC1E 6BT, UK \\ 3 Department of Chemistry, University College London, 20 Gordon St., London, WC1H 0AJ, UK \\ 4 Department of Materials, Imperial College London, Exhibition Road, London, SW7 2AZ, UK
}

Supporting Information Placeholder

\begin{abstract}
:
We present the synthesis and characterisation of a series of encapsulated diketopyrrolopyrrole red-emitting conjugated polymers. The novel materials display extremely high fluorescence quantum yields in both solution $(>70 \%)$ and thin film $(>20 \%)$. Both the absorption and emission spectra show clearer, more defined features compared to their naked counterparts demonstrating the suppression of inter and intra-molecular aggregation. We find that the encapsulation results in decreased energetic disorder and a dramatic increase in backbone co-linearity as evidenced by STM. This study paves the way for DPP to be used in emissive solid state applications and demonstrates a novel method to reduce structural disorder in conjugated polymers.
\end{abstract}

Conjugated polymers are an attractive class of fluorescent material for optical and optoelectronic applications owing to their tuneable properties through variation of chemical structure, solution processability and semi-conductivity. In condensed phases, emissive conjugated polymers are particularly sought after as they can be utilised in a wide range of applications, from organic lightemitting diodes, biomedical imaging, light emitting transistors and wavelength-tuneable lasers. It is generally understood that emission in conjugated materials arises from the radiative decay of singlet excitons. However, competing non-radiative mechanisms such as energy migration/transfer, charge separation and interchain processes such as excimer formation, present means of deactivation, thus the fluorescence quantum yield $\left(\Phi_{\mathrm{F}}\right)$ is usually reduced..$^{1}$ This is particularly evident at high concentrations (i.e. in the solid state), due to the more facile formation of aggregates. ${ }^{2}$ In the red region of the electromagnetic spectrum conjugated polymer emission is often heavily compromised, primarily due to solid state packing effects resulting from the increased planarity required to achieve narrow optical energy gaps. ${ }^{3}$ The energy-gap law further predicts reduced emissive quantum yields in the red region (and even further in the near-infrared (NIR) $)^{4-5}$, thus the development of efficient red-emitting conjugated polymers remains a key challenge. To overcome some of these issues, electronic cross-communication between adjacent polymer chains can be prevented using insulating layers that surround and isolate the $\pi$-conjugated polymer backbone, generating insulated molecular wires (IMWs). ${ }^{6-8}$ Anderson et al. pioneered the synthesis of luminescent conjugated polymers which were threaded through a sheath of non-covalently bound cyclodextrins, to form conjugated polyrotaxanes. ${ }^{9}$ Alternatively, a covalently bound approach can be taken; in 2010, Sugiyasu et al. reported on a polythiophene whose conjugated molecular wire was sheathed within its own cyclic side chains. ${ }^{10}$ It was suggested that the insulating macrocycle which was bound to adjacent monomers prevented the rotational motion of the conjugated polymer backbone, giving rise to the observed long effective conjugation lengths. More recently in 2013, Pan et al. synthesized a red-emitting IMW which achieved a rare and promising solid-state $\Phi_{\mathrm{F}}$ of $13 \% .{ }^{11}$ To our knowledge, the work exhibited by Sugiyasu and Pan present the only two examples in the literature of red-emitting IMWs which is unusual given how it could potentially overcome the solid-state quenching commonly observed in red-emitting conjugated polymers.

Diketopyrrolopyrrole (DPP) is one of the most commonly used motifs in conjugated polymer synthesis. ${ }^{12-13}$ The electron deficient and planar lactam core of DPP as well as its chemical stability and facile ability to be modified has resulted in its use in many applications, from dyes in automotive paints, to organic solar cell and transistor devices. DPP has also been used extensively in solution as a fluorescencent dye due to its high quantum yield and photostability. ${ }^{14}$ However, due to its planarity, DPP has a strong propensity to $\pi-\pi$ stack and aggregate, which becomes even more prominent in the solid state. Hence the development of DPP polymers for solid-state emissive devices has been limited to hostguest type approaches. ${ }^{15-16}$

Herein, we report the synthesis of three novel encapsulated IMWs based on DPP. Using a covalent approach, we have sheathed the DPP core using its own cyclic side chains to minimize the effects of $\pi-\pi$ stacking interactions between adjacent polymer backbones. This allows our polymers to be highly emissive, even in the solid state and we describe how this encapsulation affects the structureproperty relationships of these IMWs. The synthesis of the novel encapsulated monomer (4a) is presented in Scheme 1a. Diketofurofuran (1) was readily transformed into $N, N$-diaryl DPP (2a, $(\mathrm{R}=\mathrm{H})$ ) after reaction with aromatic amine 2,6-dimethoxyaniline in the presence of coupling reagents 4-(dimethylamino)pyridine (DMAP), 1-hydroxybenzotriazole hydrate (HOBT) and $N, N^{\prime}-$ diisopropylcarbodiimide (DIC). ${ }^{17}$ Subsequent reactions towards the synthesis of the encapsulated monomers (4a and $\mathbf{4 b}$ ) were analogous to those presented by Pan and Sugiyasu. 6,10 This involved demethylation of tetraarylated DPP (2) followed by alkyla- 
tion with 1-bromopentene to afford compound (3). The product was then subjected double ring-closing olefin metathesis and hydrogenated using $\mathrm{Pd} / \mathrm{C}(10 \mathrm{~mol} \%)$ to form the encapsulated monomer (4a) in a $44 \%$ isolated yield after column chromatography. (see S1)

Scheme 1: a) Synthesis of encapsulated monomers (4a) and (4b) from DFF (1); b) Encapsulated polymers c) Naked

a)<smiles>O=C1OC(c2ccc(Br)cc2)C2C(=O)OC(c3ccc(Br)cc3)=C12</smiles>

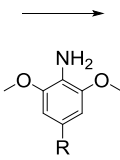

1

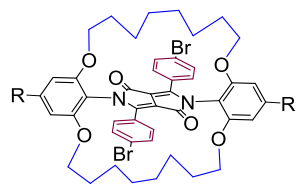

4a: $\mathrm{R}=\mathrm{H}$

4b: $\mathrm{R}=\mathrm{C}_{13} \mathrm{H}_{27}$

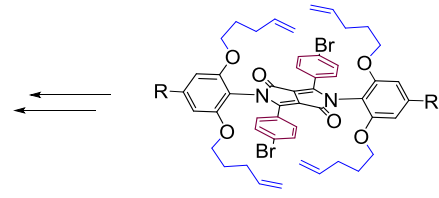

3

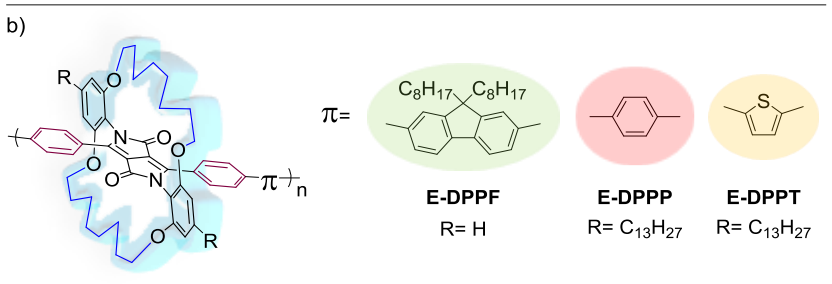

c)

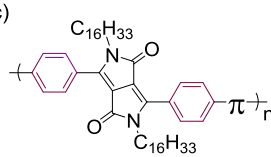

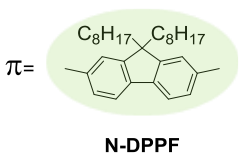

$\mathrm{R}=\mathrm{H}$

N-DPPP N-DPPT

polymers

The structure of the encapsulated monomer was determined by Xray crystallographic analysis (single crystal grown from chloroform) which confirmed sheathing of the DPP core rather than encapsulation of the amines (Figure 1). The alkylated derivative (4b) was prepared using an identical method using 2,6dimethoxy-4-tridecylaniline in place of 2,6-dimethoxyaniline (S4). The encapsulated monomer (4a) was then copolymerised with a diborylated fluorene derivative in a Suzuki cross-coupling reaction to form the novel encapsulated polymer E-DPPF. We sought to reduce the distance between the encapsulated monomers thereby increasing the density of encapsulation within our polymers. This led us to look at phenyl and thiophene as alternatives to fluorene.

\section{Figure 1: Crystal structure of encapsulated DPP monomer (4a)}

Polymer E-DPPP was synthesised by coupling 1,4benzenediboronic acid bis(pinacol)ester with our encapsulated monomer (4b) in a Suzuki cross coupling reaction and polymer $\mathbf{E}$ DPPT was synthesised from a Stille cross-coupling reaction of (4b) with 2,5-bis(trimethylstannyl) thiophene. In addition to the encapsulated polymers (Scheme 1b) the naked counterparts $\mathbf{N}$ DPPF, N-DPPP and N-DPPT were also synthesised for comparison (Scheme 1c), thus allowing us to understand how molecular encapsulation affects the photophysical properties of conjugated materials. The polymers were purified by Soxhlet extraction using acetone then hexane, to remove low molecular weight oligomers, followed by chloroform.

\section{Table 1: Physical properties of novel polymers}

\begin{tabular}{|l|l|l|l|}
\hline Polymer & $\mathrm{M}_{\mathrm{n}}{ }^{\mathrm{a}}(\mathrm{kDa})$ & $\mathrm{M}_{\mathrm{w}}{ }^{\mathrm{a}}(\mathrm{kDa})$ & $\mathrm{PDI}^{a}$ \\
\hline E-DPPF & 28.7 & 65.8 & 2.29 \\
\hline E-DPPP & 20.2 & 26.7 & 1.32 \\
\hline E-DPPT & 19.7 & 23.8 & 1.21 \\
\hline N-DPPF & 3.8 & 7.7 & 2.03 \\
\hline N-DPPP & 16.9 & 47.4 & 2.89 \\
\hline N-DPPT & 41.8 & 65.1 & 1.56 \\
\hline
\end{tabular}

${ }^{a}$ Determined by GPC (CB) against PS standards;

Interestingly, despite multiple attempts it was not possible to successfully synthesize N-DPPF in reasonable molecular weight despite it having the largest amount of alkyl chain density. Due to its unsuitably low molecular weight $\mathbf{N}$-DPPF was discounted from further study. With the exception of N-DPPF all polymers were synthesized in reasonable molecular weights and polydispersities (Table 1) and displayed good solubility in chlorinated solvents. Figure 2 shows the solution and thin film absorption and emission spectra of the encapsulated $(\mathrm{E})$ and naked $(\mathrm{N})$ polymers synthesised. The absorption and emission profiles of all polymers show a bathochromic shift from solution to thin film (Table 2), attributed to planarization that arises in the solid state. For EDPPF the solution absorption and emission profiles show an absorption maxima at $\sim 528 \mathrm{~nm}$ and a slight shoulder which we attribute to vibronic coupling at shorter wavelengths $(\sim 510 \mathrm{~nm})$. The emission in solution clearly shows the vibronic fine structure with the emission maxima from the $0-0$ transition at $571 \mathrm{~nm}$. Going from solution to thin film, the polymer appears to retain some of its vibronic structure in the absorption spectrum. The vibrational fine structure in emission can be observed with the 0,1 transition being increased in intensity. In the case of the encapsulated phenyl derivative E-DPPP a similar set of features are observed. In solution, the absorption has a maxima at $\sim 532 \mathrm{~nm}$ with a shoulder at $\sim 500 \mathrm{~nm}$. The emission again shows clear fine structure with a maxima at $\sim 572 \mathrm{~nm}$. In comparison the naked polymer N-DPPP shows very different features. The absorption in solution displays a much shallower onset and a blue-shifted absorption maxima with no evidence of any fine structure. The emission spectrum of N-DPPP is very broad and red-shifted, although there appears to be some evidence of the 0-0 emissive transition. In the solid state the E-DPPP absorption spectrum displays a slightly more prominent shoulder than in solution, and the emission spectrum shows fine structured emission with a red-shifted maximum corresponding to the 0-1 transition. In contrast, N-DPPP shows a broad featureless emission spectrum with no fine structure reminiscent of excimeric or other aggregate emission. The encapsulated thiophene containing polymer E-DPPT has a red-shifted absorption maxima $(581 \mathrm{~nm})$ due to the enhanced planarity with a shoulder at $\sim 550 \mathrm{~nm}$ which is enhanced in the solid state. The emission of E-DPPT shows narrow fine-structured emission in both solution and solid state with a maximum at $621 \mathrm{~nm}$.
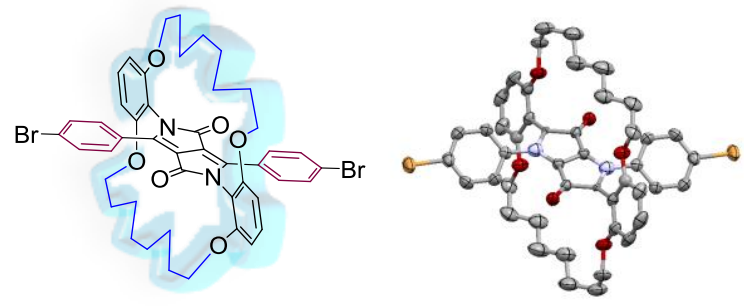

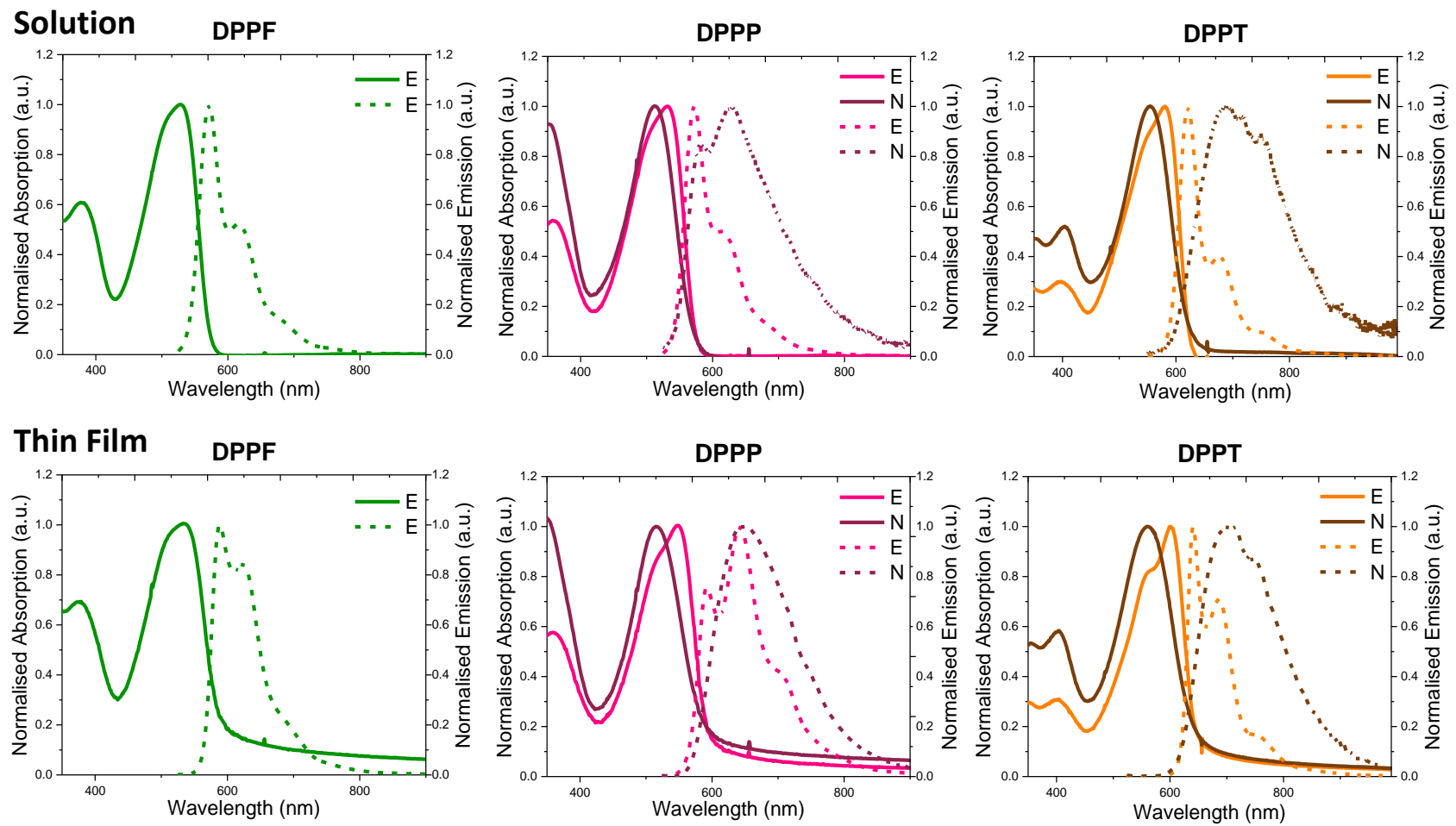

Figure 2: Normalised solution $(\mathrm{CB} \sim 4 \mu \mathrm{g} / \mathrm{mL}$ ) and thin film (spin-coated from $\mathrm{CB} 10 \mathrm{mg} / \mathrm{mL}$ ) absorption and emission spectra of polymers

In comparison, its naked equivalent $\mathbf{N}$-DPPT shows a more featureless, blue-shifted absorption in both solution and solid state and a significantly red-shifted, broad featureless emission. The steeper, more well-defined absorption spectra of the encapsulated polymers and the sharper emission peaks suggests a lower degree of energetic disorder than their naked counterparts. ${ }^{18-19}$ Sugiyasu et al. reported a similar effect which was attributed to their macrocycle enforcing rigidity across two adjacent thiophenes. ${ }^{10}$ However, in our case the macrocycle does not span adjacent units so this is not possible. Such structural rigidification is typically only observed on going from solution to solid state (where stacking effects introduce greater ordering), thus our reduced relative disorder in solution is highly unusual. The red-shifted broad emission in solution from the naked polymers indicates that considerable intra-molecular aggregation is occurring, which has clearly been completely suppressed through encapsulation. It is also evident that the aggregate (either inter or intra) emission observed in the solid state for the naked polymers is largely suppressed through our encapsulation strategy.

Table 2: Optical properties of novel polymers

\begin{tabular}{|l|l|l|l|l|}
\hline Polymer & $\begin{array}{l}\text { Emission } \\
\max _{\text {soln. }^{a}}\end{array}$ & $\begin{array}{l}\text { Emission } \\
\max _{\text {film }^{b}}\end{array}$ & $\begin{array}{l}\Phi_{\mathrm{F}} \quad \text { (Solu- }_{\text {tion) }}{ }^{c} \\
(\%)\end{array}$ & $\begin{array}{l}\Phi_{\mathrm{F}\left(\text { Film}^{c}\right.}{ }^{c} \\
(\%)\end{array}$ \\
\hline E-DPPF & 571 & 589 & $94.8 \pm 1.0$ & $19.8 \pm 1.1$ \\
\hline E-DPPP & 572 & 641 & $74.0 \pm 1.2$ & $22.6 \pm 1.1$ \\
\hline E-DPPT & 621 & 641 & $73.6 \pm 1.2$ & $27.8 \pm 1.3$ \\
\hline N-DPPF & & - & - & - \\
\hline N-DPPP & 629 & 644 & $19.0 \pm 3.3$ & $8.0 \pm 0.3$ \\
\hline N-DPPT & 686 & 703 & $18.2 \pm 1.3$ & $5.6 \pm 0.5$ \\
\hline
\end{tabular}

${ }^{a} \mathrm{CB}$ solution $(\sim 4 \mu \mathrm{g} / \mathrm{mL}) ;{ }^{b}$ Spin-coated from $\mathrm{CB}(10 \mathrm{mg} / \mathrm{mL})$; ${ }^{c}$ Measured using an integrating sphere.

This interpretation is strongly supported by the photoluminescence quantum yields (PLQY). In solution, the encapsulated polymers display extremely high fluorescence quantum yields (>70\%) with a particularly remarkable $\Phi_{\mathrm{F}} 95 \%$ for E-DPPF. In contrast, the naked polymers show significant PL quenching $\left(\Phi_{\mathrm{F}}\right.$ $\sim 20 \%$ ) further suggesting that intra-molecular aggregation is occurring. In the solid state, the emission of the naked polymers is further quenched to $<10 \%$ PLQY. Most importantly, the encapsulated polymers retain impressive PLQY in the solid state $(\sim 20 \%)$. In particular E-DPPT displays a thin film fluorescent quantum yield of $\sim 28 \%$ which is amongst the highest value reported for a neat conjugated polymer in this wavelength region. We note that these findings are in direct contradiction to the recent study which suggests that polymers with higher energetic disorder should have higher emissive quantum yields. ${ }^{20} \mathrm{We}$ suggest that the introduction of the encapsulating macrocycle prevents the formation of non-radiative quenching sites. The emissive quantum yields clearly demonstrate our encapsulation strategy has been highly effective, resulting in the first examples of highly fluorescent DPP polymers in the solid state potentially allowing for their use in sensors, LEDs and visible light communications (VLC) applications. $^{21}$

In order to understand the unusual apparent decrease in energetic disorder the DPPP and DPPT polymers were imaged using scanning tunnelling microscopy (STM). Figure 3 displays the encapsulated polymers alongside their naked equivalents. All four polymers display lamellar type packing into nanostructured domains. However, there is a stark difference between the encapsulated and naked polymers. E-DPPP and E-DPPT show extremely low defect domains where there are virtually no twisted or bent polymer strands. E-DPPT in particular shows effectively defect free polymer domains where each strand is in a completely linear confor- 
mation. In contrast N-DPPP and N-DPPT show typical coiled and worm-like nanostructuring, characteristic of other polymers imaged by STM with numerous 'hairpin' like conformational defects. $^{22}$
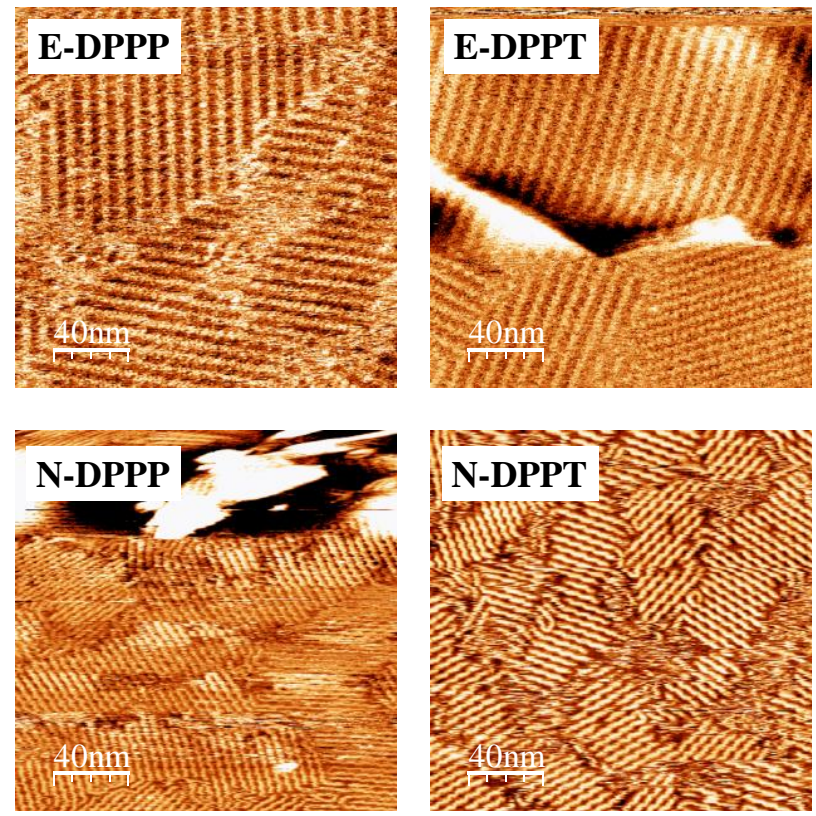

Figure 3. STM images of conjugated polymers (E-DPPP: sample bias (Vs) of $-0.50 \mathrm{~V}$ with respect to the tip and a tunnelling current of (It) of $5.0 \mathrm{pA}$; E-DPPT: Vs $=-0.55 \mathrm{~V}$, It $=5.0 \mathrm{pA}$; $\mathbf{N}$ DPPP: Vs $=-0.85 \mathrm{~V}$, It $=7.5 \mathrm{pA}$; N-DPPT: Vs $=-0.70 \mathrm{~V}$, It $=7.0$ pA.)

Given the similarity of the solution and thin film UV-Vis absorption spectra we believe that these encapsulated polymers therefore have a general tendency to adopt highly linear conformations in both solution and solid state. To investigate this we studied the materials with molecular modelling. Representative symmetric oligomers were constructed from fragments of two DPP units, and three hydrogen end-capped donors. Molecular dynamics was then simulated for these oligomers in vacuum, using the PM6 semiempirical method. ${ }^{23}$ The molecular dynamics was simulated with a time step of $1 \mathrm{fs}$, for a total time of $20 \mathrm{ps}$, with a thermostat at $300 \mathrm{~K}$. Snapshots of these trajectories were extracted from every $0.5 \mathrm{ps}$ (a total of 40 frames). These structures from the thermodynamic ensemble were then used as the input for an excited state calculation. At the Zindo/S theory level, it is observed that the encapsulated structures have considerably large band gap fluctuations. (Sampled standard deviations of E-DPPF: $112 \mathrm{meV}, \mathbf{E}-$ DPPP: $185 \mathrm{meV}$, E-DPPT: $137 \mathrm{meV}$ ). At the oligomer level these band-gap fluctuations are considerably larger than has been calculated for typical non-encapsulated materials. ${ }^{24}$ This suggests that the observed reduction in energetic disorder for the encapsulated form is due to longer-range effects than just considering the torsions of these small oligomers. We believe that this increased ordering is from the 3-dimensional steric shielding effect from the encapsulating unit which prevents bending of the polymer backbone. Disorder-free charge transport in conjugated polymers has recently been observed but the synthetic guidelines for reducing energetic disorder are not clear. ${ }^{25}$ Thus polymer encapsulation offers a novel method for increasing backbone linearity in both solution and solid state.

In conclusion we have synthesized a series of encapsulated conjugated diketopyrrolopyrrole polymers. These materials display extremely high fluorescence quantum yields in both solution and thin film allowing this ubiquitous chromophore to be used, for the first time, as an emissive material in the solid state. The encapsulated polymers display much sharper emissive spectral features than their naked counterparts demonstrating that both intra and intermolecular aggregation has been suppressed. Furthermore, the 3-dimensional encapsulating steric shield promotes enhanced backbone co-linearity resulting in sharper absorption features in both solution and thin film. STM imaging of the encapsulated materials showed remarkably linear, conformationally defect-free polymer domains. Therefore we believe that our encapsulated DPP polymers are ideal candidates for many next generation optical and optoelectronic applications where control of emission, aggregation and polymer conformation is key.

\section{ASSOCIATED CONTENT}

\section{Supporting Information}

The Supporting Information is available free of charge on the ACS Publications website.

Synthesis and characterization of polymers, crystallographic data and additional theoretical calculations.

\section{Accession Codes}

CCDC 1565490 and 1565494 contains the supplementary crystallographic data for this paper. These data can be obtained free of charge via www.ccdc.cam.ac.uk/data_request/cif, or by emailing data_request@ccdc.cam.ac.uk, or by contacting The Cambridge Crystallographic Data Centre, 12, Union Road, Cambridge CB2 1EZ, UK; fax: +44 1223336033.

\section{AUTHOR INFORMATION}

\section{Corresponding Author}

hab60@cam.ac.uk

\section{Author Contributions}

†These authors contributed equally.

\section{Notes}

The authors declare no competing financial interests.

\section{ACKNOWLEDGMENT}

This work was funded by EU project 679789 - 455 CONTREX. This work was also supported by the EU H2020 ETN SYNCHRONICS under grant agreement 643238. FC is a Royal Society Wolfson Research Merit Award holder.

\section{REFERENCES}

(1) McQuade, D. T.; Kim, J.; Swager, T. M., J. Am. Chem. Soc. 2000, 122,5885 .

(2) Hu, Z.; Willard, A. P.; Ono, R. J.; Bielawski, C. W.; Rossky, P J.; Vanden Bout, D. A., Nat. Commun. 2015, 6, 8246.

(3) Harkin, D. J.; Broch, K.; Schreck, M.; Ceymann, H.; Stoy, A.; Yong, C.-K.; Nikolka, M.; McCulloch, I.; Stingelin, N.; Lambert, C.; Sirringhaus, H., Adv. Mater. 2016, 28, 6378.

(4) Wilson, J. S.; Chawdhury, N.; Al-Mandhary, M. R. A.; Younus, M.; Khan, M. S.; Raithby, P. R.; Köhler, A.; Friend, R. H., J. Am. Chem. Soc. 2001, 123, 9412.

(5) Zampetti, A.; Minotto, A.; Squeo, B. M.; Gregoriou, V. G.; Allard, S.; Scherf, U.; Chochos, C. L.; Cacialli, F., Sci. Rep. 2017, 7, 1611 .

(6) Pan, C.; Zhao, C.; Takeuchi, M.; Sugiyasu, K., Chem. Asian J. 2015, 10, 1820. 
(7) Frampton, M. J.; Anderson, H. L., Angew. Chem. Int. Ed. 2007, 46, 1028.

(8) Terao, J.; Tanaka, Y.; Tsuda, S.; Kambe, N.; Taniguchi, M.; Kawai, T.; Saeki, A.; Seki, S., J. Am. Chem. Soc. 2009, 131, 18046.

(9) Cacialli, F.; Wilson, J. S.; Michels, J. J.; Daniel, C.; Silva, C.; Friend, R. H.; Severin, N.; Samorì, P.; Rabe, J. P.; O'Connell, M. J.; Taylor, P. N.; Anderson, H. L., Nat. Mater. 2002, 1, 160.

(10) Sugiyasu, K.; Honsho, Y.; Harrison, R. M.; Sato, A.; Yasuda T.; Seki, S.; Takeuchi, M., J. Am. Chem. Soc. 2010, 132, 14754.

(11) Pan, C.; Sugiyasu, K.; Wakayama, Y.; Sato, A.; Takeuchi, M., Angew. Chem. Int. Ed. 2013, 52, 10775.

(12) Bronstein, H.; Chen, Z.; Ashraf, R. S.; Zhang, W.; Du, J.; Durrant, J. R.; Shakya Tuladhar, P.; Song, K.; Watkins, S. E.; Geerts, Y.; Wienk, M. M.; Janssen, R. A. J.; Anthopoulos, T.; Sirringhaus, H.; Heeney, M.; McCulloch, I., J. Am. Chem. Soc. 2011, 133, 3272.

(13) Nielsen, C. B.; Turbiez, M.; McCulloch, I., Adv. Mater. 2013 $25,1859$.

(14) Kaur, M.; Choi, D. H., Chem. Soc. Rev. 2015, 44, 58.

(15) Data, P.; Kurowska, A.; Pluczyk, S.; Zassowski, P.; Pander, P.; Jedrysiak, R.; Czwartosz, M.; Otulakowski, L.; Suwinski, J.; Lapkowski, M.; Monkman, A. P., .J. Phys. Chem. C 2016, 120, 2070.

(16) Fenwick, O.; Fusco, S.; Baig, T. N.; Stasio, F. D.; Steckler, T. T.; Henriksson, P.; Fléchon, C.; Andersson, M. R.; Cacialli, F., APL Materials 2013, 1, 032108 .

(17) Riggs, R. L.; Morton, C. J. H.; Slawin, A. M. Z.; Smith, D. M.; Westwood, N. J.; Austen, W. S. D.; Stuart, K. E., Tetrahedron 2005, 61, 11230 .

(18) Panzer, F.; Bässler, H.; Köhler, A., J. Phys. Chem. Lett. 2017 8,114 .

(19) Barford, W.; Marcus, M., J. Chem. Phys. 2017, 146, 130902.

(20) Rörich, I.; Mikhnenko, O. V.; Gehrig, D.; Blom, P. W. M.; Crăciun, N. I., J. Phys. Chem. B 2017, 121, 1405.

(21) Haigh, P.A.; Bausi, F.; Kanesan, T.; Le, S. T.; Rajbhandari, S. Ghassemlooy, Z.; Papa-konstantinou, I.; Popoola, W.; Burton, A.; Le Minh, H.; Ng, W. P.; Ellis, A. D.; Cacialli, F., IEEE Photonics Technology Letters 2014, 26, 1975

(22) Xu, L.; Yang, L.; Lei, S., Nanoscale 2012, 4, 4399.

(23) Stewart, J. J. P., J. Mol. Model. 2007, 13, 1173.

(24) Frost, J. M.; Kirkpatrick, J.; Kirchartz, T.; Nelson, J., Faraday Discuss. 2014, 174, 255.

(25) Venkateshvaran, D.; Nikolka, M.; Sadhanala, A.; Lemaur, V.; Zelazny, M.; Kepa, M.; Hurhangee, M.; Kronemeijer, A. J.; Pecunia, V.; Nasrallah, I.; Romanov, I.; Broch, K.; McCulloch, I.; Emin, D.; Olivier, Y.; Cornil, J.; Beljonne, D.; Sirringhaus, H., Nature 2014, 515, 384

\section{Table of Contents artwork}

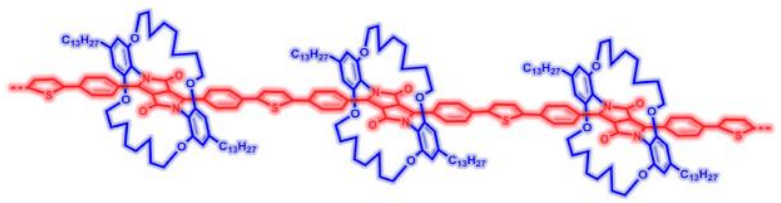


